\title{
Gas Chromatographic Fid, Hypoglycemic and Hypolipidemic Effects Of Leaves Of Laportea Aestuans In Alloxan Induced Diabetes In Male Albino Rats
}

\author{
${ }^{1}$ Okereke, S.C*.. ${ }^{1}$ Elekwa, I and ${ }^{2}$ Nmaju, A.U \\ ${ }^{I}$ Department of Biochemistry, Abia State University, Uturu, Nigeria. \\ ${ }^{2}$ Department of Physiology, Abia State University, Uturu
}

\begin{abstract}
Gas chromatophic FID analysis of leaves of Laportea aestuans was conducted and showed the presence of polyphenols, flavonoids, terpenes, carotenoids and saponins. The effects of Laportea (Linn) on alloxan induced diabetes in rats were also evaluated using glucose and lipid profiles. Animals weighing (160 $200 \mathrm{~g}$ ) were divided into 3 groups of 21 animals each; Group 1 (normal control), Group 2 (diabetes control) and Group 3 (test). Diabetes was induced in albino rats by intraperitoneal injection of alloxan in group 2 and 3 respectively. Animals in group 1 and 2 received normal rat feed while animals in group 3 were fed with Laportea aestuans leaves for a period of 28 days. Animals were sacrificed on day 28. Glucose, glycated hemoglobin and lipid profile were estimated. Laportea aestuans leaves elicited significant $(P<0.05)$ reduction of blood glucose and lipid profile parameters except high density lipoprotein cholesterol (HDL-C) which significantly increased. L. aestuans offer
\end{abstract} promise as antidiabetic and antihyperlipidemia.

Key words: Gas - chromatography - FID, Laportea aestuans, diabetes, hypercholesterolemia

\section{Introduction}

Man's dependence on plants for shelter, clothing, food, flavours and fragrances has been documented. Plants form the basis for traditional medicine which has given rise to some important drugs in use today [1]. As more people come to adopt traditional herbal medicine as alternative health care system, there is need for more scrutiny on different plants in the treatment and prevention of disease, especially the chronic diseases.

Arteriosclerosis or coronary heart disease is a condition characterized by deposition of lipids mainly cholesterol on the endothelium of the arteries. These deposits narrow arterial channels and partly block the normal flow of blood through them [2]. Stroke, partial paralysis, loss of speech and in some cases death, may arise due to the decreased blood flow and oxygen [3]. Arteriosclerosis is becoming more prevalent in the developing countries, as it is in the developed countries [4].

Plant foods including fruits, nuts, seeds and vegetables largely contribute to the local diet in developing countries [5]. Staple food, flavours, spices and beverages play a role in the nutrition and health of their consumers [6]. One of such common traditional plant foods found in West Africa, though not popularly eaten as vegetable is Laportea aestuans (Stinging nettle).

Laportea aestuans is an annual herb of the nettle family. It is possibly native of tropical Africa, although it is now wide spread. It can be found in sub - tropical regions like California, Florida, Puertoico, Central America, the West Indies and India [7].

L. aestuans is widely used in African traditional medicine. The pulped whole plant is eaten or the plant sap is drunk as an antihelmintic and for the treatment of hernia. Pulp of the plant is rubbed into scarofication to cure headache and syphilitic yaws. Plant extract is drank against cough or rubbed on the body to treat fever. $L$. aestuans is used to treat gonorrhea. An infusion of the leaf is taken for the treatment of rheumatism and menopausal disorders. A decoction of the leaf and root is drunk as an antidote to any case of poisoning [1]. It is believed that $L$. aestuans as a vegetable has potential chemopreventive properties among other uses. The aim of this research paper is to establish the hypoglycermic and hypolipidemic effects of leaves of Laportea aestuans.

Materials

\section{Materials And Methods}

The leaves of Laportea aestuans (Stinging nettle) were harvested fresh from the botanic garden of Abia State University, Uturu, Nigeria and identified by Dr. S.K Chukwuka of the Plant Science Department of Abia State Univeristy. Voucher Specimen was deposited in the herbarium of the Abia State University (Voucher no: ABSU 125). 


\section{Chemicals and Reagents}

- Alloxan monohydrate Sigma Aldrich MO, USA.

- One touch accucheck glucometer (Roche Diagnostic Germany).

- Commercial kit for glycated hemoglobin (HBALc) Randox Laboratory UK.

- Total plasma cholesterol (TC), triglyceride (TG), High density lipoprotein cholesterol (HDL-C) and low density lipoprotein cholesterol (LDL-C) kit were used (Biolab SA Maizy, France).

\section{Animal Model}

Sixty three (63) male albino rats were used to perform the study. The rats weighed between $160-200 \mathrm{~g}$. They were purchased from the animal farm of University of Nigeria, Nsukka.

The animals were house in plastic cages and placed on commercial rat feed. They were allowed to feed and water ad libitum for a week to acclimatize prior to commencement of study. The animals were randomly divided into three groups of 21 animals each. Group 1 served as the control group and received rat feed. Diabetes was induced in group 2 and 3 using a single dose of $120 \mathrm{mg} / \mathrm{Kg}$ body weight of alloxan monohydrate. Group 2 received rat feed with group 1, while group 3 was fed rat feed supplemented with 50\% w/w leaves of Laportea aestuans. All the animals were allowed feed and water ad libitum for 28 days.

\section{Collection of Blood Samples from Rats}

Rats were anasthetized with chloroform at the end of 28 days and sacrificed by cutting through the jugular vein. Blood was collected in clean dry beaker and allowed to cloth. It was centrifuged at 3000rpm for 10 minutes; the supernatant (serum) was collected and stored in the refrigerator for use.

\section{Statistical Analysis}

All values were represented as mean $\pm \mathrm{SD}$ and subjected to statistical analysis using GraphPad Prism (Version 6.0) software. Comparison was done using onw - way analysis of variance (ANOVA). Values were considered significant when $\mathrm{P}<0.05$.

\section{Results And Discussion}

The results of the Gas - chromatographic analysis identified the various compounds present in the plant as shown in tables 1 to 5 . The compounds were identified through the NIST08L database.

Table 1:Phenolic compounds identified in the methanolic leaf extract of Laportea aestuans showing their retention time (RT) and amount $(\mathbf{m g} / \mathbf{1 0 0 g})$.

\begin{tabular}{lll}
\hline Name & Retention time (min) & Amount $(\mathbf{m g} / \mathbf{1 0 0 g})$ \\
\hline Vanilic acid & 15.160 & 5.923 \\
Gallic acid & 16.040 & 4.209 \\
Ferulic acid & 18.055 & 4.738 \\
(6) - Gingerol + capsaicin & 19.627 & 1.545 \\
Rosemarnic acid & 20.594 & 1.115 \\
Tannic acid & 22.507 & 122.613 \\
P - Coumaric acid & 11.374 & 8.741 \\
Caffeic acid & 14.362 & 3.411 \\
Scopoletin & 16.659 & 4.050 \\
\hline
\end{tabular}

Table 2: Flavonoids identified in the methanolic leaf extract of Laportea aestuans showing their retention time (RT) and amount ( $\mathrm{mg} / \mathbf{1 0 0 g})$.

\begin{tabular}{lcc}
\hline Name & $\begin{array}{c}\text { Retention } \\
\text { time (min) }\end{array}$ & Amount (mg/100g) \\
\hline Catechin & & 13.738 \\
Resveratrol & 15.039 & 2.051 \\
Genistein & 15.498 & 5.643 \\
Apigenin & 16.034 & 5.487 \\
Kaemferol & 18.048 & 5.715 \\
(-)-Epicatechin & 19.514 & 9.365 \\
(-)-Epigallocatechin & 20.467 & 3.469 \\
Ellagic acid & 24.417 & 8.121 \\
Myricetin & 24.786 & 6.083 \\
Quercetin-3,7,4-trimethyl ether & 26.061 & 1.059 \\
Quercetin-3,7,3',4'-trimethyl ether & 26.732 & 1.274 \\
Artemetin & 26.824 & 3.716 \\
\hline
\end{tabular}


Gas Chromatographic Fid, Hypoglycemic And Hypolipidemic Effects Of Leaves Of Laportea

\begin{tabular}{lll}
\hline Kaempferol-arabinoside & & 4.040 \\
Quercitrin & 27.284 & 7.834 \\
Isoquercetin & 27.424 & 2.563 \\
Naringin & 27.799 & 2.364 \\
Rutin & 27.907 & 2.413 \\
Hesperidin & 28.094 & 1.841 \\
\hline
\end{tabular}

Table 3: Terpenes identified in the chloroform leaf extract of Laportea aestuans showing their retention time (RT) and amount $(\mathrm{mg} / \mathbf{1 0 0 g})$.

\begin{tabular}{|c|c|c|}
\hline Name & $\begin{array}{c}\text { Retention } \\
\text { time } \\
\text { (min) }\end{array}$ & Amount (mg/100g) \\
\hline Limonene & 9.318 & 29.248 \\
\hline Alpha Pinene & 9.669 & 9.558 \\
\hline Beta Pinene & 10.848 & 13.634 \\
\hline Cis Ocimene & 12.268 & 9.905 \\
\hline Myrcene & 12.739 & 13.608 \\
\hline Citronellol & 19.236 & 23.183 \\
\hline Neryl Acetate & 21.501 & 10.012 \\
\hline
\end{tabular}

Table 4: Carotenoids identified in the acetone leaf extract of Laportea aestuans showing their retention time (RT) and amount $(\mathrm{mg} / \mathbf{1 0 0 g})$.

\begin{tabular}{|c|c|c|}
\hline Name & $\begin{array}{l}\text { Retention } \\
\text { time } \\
\text { (min) }\end{array}$ & Amount (mg/100g) \\
\hline Malvidine & 19.029 & 8.634 \\
\hline Lycopene & 21.330 & 7.574 \\
\hline Carotene & 22.606 & 20.121 \\
\hline Lutein & 23.234 & 2.444 \\
\hline Xanthophyll & 24.032 & 1.120 \\
\hline Anther - xanthine & 24.881 & 5.389 \\
\hline Asta - xanthine & 25.619 & 9.821 \\
\hline Viola - xanthine & 26.357 & 9.228 \\
\hline Neo - xanthine & 26.997 & 1.338 \\
\hline
\end{tabular}

Table 5: Saponins identified in the methanolic leaf extract of Laportea aestuans showing their retention time (RT) and amount $(\mathrm{mg} / \mathbf{1 0 0 g})$.

\begin{tabular}{lcc}
\hline Name & $\begin{array}{c}\text { Retention } \\
\text { time (min) }\end{array}$ & Amount (mg/100g) \\
\hline Hispogenin & 17.355 & 2.048 \\
Diosgenin & 19.511 & 3.199 \\
Neochlorogenin & 20.467 & 7.122 \\
Hecogenin & 21.433 & 1.747 \\
Saponine & 26.280 & 2.873
\end{tabular}

The effects of Laportea aestuans supplemented diet on the blood glucose, glycated hemoglobin and lipid profile in normal and alloxan induced diabetes in male rats were shown in tables 6 and 7.

Table 6: Effects of Laportea aetuans on glucose (mg/dl) and glycated hemoglobin level (\%) in normal and alloxan induced diabetes in male rats.

\begin{tabular}{|c|c|c|c|c|c|c|}
\hline \multicolumn{6}{|c|}{ Glucose } & \multirow{2}{*}{$\begin{array}{l}\text { Glycated } \\
\text { Hemoglobin on day } 28\end{array}$} \\
\hline & 0 & 7 & 14 & 21 & 28 & \\
\hline \multirow{2}{*}{ Group 1} & 98.00 & 104.00 & 96.90 & 101.00 & 102.20 & $3.97 \pm 0.009^{c}$ \\
\hline & $\pm 1.4^{\mathrm{c}}$ & $\pm 1.30^{\mathrm{c}}$ & $\pm 0.66^{\mathrm{c}}$ & $\pm 0.67^{\mathrm{c}}$ & $\pm 1.06^{\mathrm{c}}$ & \\
\hline \multirow[t]{2}{*}{ Group 2} & 272.10 & 191.20 & 163.10 & 158.00 & 158.30 & $9.85 \pm 0.52^{\mathrm{a}}$ \\
\hline & $\pm 0.76^{\mathrm{a}}$ & $\pm 0.33^{\mathrm{a}}$ & $\pm 0.72^{\mathrm{a}}$ & $\pm 0.77^{\mathrm{a}}$ & $\pm 0.76^{\mathrm{a}}$ & \\
\hline \multirow[t]{2}{*}{ Group 3} & 269.30 & 162.30 & 133.20 & 233.10 & 122.30 & $5.46 \pm 0.37^{\mathrm{b}}$ \\
\hline & $\pm 1.22^{\mathrm{b}}$ & $\pm 0.72^{\mathrm{b}}$ & $\pm 0.82^{\mathrm{b}}$ & $\pm 0.72^{\mathrm{b}}$ & $\pm 0.48^{\mathrm{b}}$ & \\
\hline
\end{tabular}

Values with different alphabetical superscription in a row are significantly different al $\mathrm{P}<0.05$

Data are mean of three rats in each group \pm SD 
Table 2: Effect of Laporteaaestuans on serum lipid profile (mg/dl) in normal and alloxan induced diabetes in male albino rats (day 28).

\begin{tabular}{llllll}
\hline & LDL & HDL & VLDL & TG & \multicolumn{1}{c}{ TC } \\
\hline Group 1 & 35.70 & 33.40 & 13.33 & 53.45 & 70.43 \\
& $\pm 2.29^{\mathrm{b}}$ & $\pm 0.65^{\mathrm{C}}$ & $\pm 0.35^{\mathrm{b}}$ & $\pm 1.98^{\mathrm{b}}$ & $\pm 1.28^{\mathrm{b}}$ \\
& & & & \\
Group 2 & 105.51 & 39.38 & 33.14 & 55.33 & 174.22 \\
& $\pm 3.98^{\mathrm{a}}$ & $\pm 0.30^{\mathrm{b}}$ & $\pm 0.38^{\mathrm{a}}$ & $\pm 4.86^{\mathrm{a}}$ & $\pm 3.05^{\mathrm{a}}$ \\
Group 3 & 19.23 & 47.58 & 14.60 & 64.81 & 77.33 \\
& $\pm 1.56^{\mathrm{c}}$ & $\pm 0.34^{\mathrm{a}}$ & $\pm 1.25^{\mathrm{b}}$ & $\pm 4.98^{\mathrm{b}}$ & $\pm 0.88^{\mathrm{b}}$ \\
\hline
\end{tabular}

Values with different alphabetical superscription in a row are significantly different al $\mathrm{P}<0.05$

Data are mean of three rats in each group \pm SD

\section{Discussion}

Phytochemicals such as phytosterol, carotenoids, lycopenes, terpenes, flavonoids, coumarin, quercetin, limonene etc; are known to produce chemopreventive effects on animal models. These phytochemicals are reported to have anticarcinogenic and cardiovascular effects. This study showed that the plant food Laportea aestuans contained phytochemicals that have chemopreventive effects.

Diabetes mellitus remains the most common chronic disorder of carbohydrate, fat and protein metabolism. It is characterized by chronic and persistent hyperglycemia, degenerative vascular changes and neuropathy due to complete or partial insulin secretion or insulin resistance [8].

Apart from hyperglycemia, diabetes mellitus is accompanied by hypercholesterolemia and hepatic steatosis. Hypercholesterolemia is the consequence of accelerated fatty acid oxidation to acetyl CoA which is the primary substance of cholesterol synthesis. Similarly, the hyperlipidemia associated with diabetes mellitus results from accerated de novo hepatic biosynthesis and relaease of VLDL-C without a corresponding increase in the rate of clearance from the blood by the lipoprotein lipase whose activity is dependent in high insulin: glucagon ratio [9].

Diabetes hyperglycemia results in an increase in free-radical production by a mechanism involving glucose oxidation followed by protein glycation and oxidative degeneration. Glycation (non-enzymatic glycosylation) involves the condensation of glucose with the $\alpha$-amino group of an N-terminal amino acid or the amines of nucleic acid. Formation of advanced glycation end products (AGEs) are believed to be involved in the genesis of many of the irreversible complications of diabetes, including expanded extracellular matrix, cellular hypertrophy hyperplasia and vascular complications. Marker used for estimating the degree of protein glycation in diabetes includes fructosamine and glycated hemoglobin levels [10].

There was a significant reduction $(\mathrm{P}<0.05)$ in serum glucose for the test group that was fed with Laportea aestuans leaves supplemented diet, when compared to the diabetes induced group and the normal control group. From this study, it showed that diabetic albino rat fed with L. aestuans leaves for as long as four (4) weeks led to reduced glycemia $(\mathrm{P}<0.05)$. [11,] demonstrated similar hypoglycemic effect using Semecarpus anacadium Linn on streptozotocin induced diabetes in rats.

This L. aestuans leaves may probably contain active substances that possess blood glucose lowering activity [12]. It was also observed iin the study that there was a significant reduction of glycated hemoglobin in the test group when compared with the induced control (diabetic rats) and normal control rats $(\mathrm{P}<0.05)$. It is therefore possible that the leaves of $L$. aestuans may possess active substances which scavenge the free radicals of glucose oxidation, protein glycation and oxidative degeneration or probably an improvement in insulin secretion. [13], demostrated that Trigonella foenumgraecum (Fenugreek) seeds show improved glycemic control (significant decrease) in glycated hemoglobin (HBAIc) on day 28 treatments as compared with streptozotocin (n-STZ) control rats.

This study also showed a decrease in LDL, VLDL, TG and TC in the test group (Group 3) when compared with the induced (Group 2) at $(\mathrm{P}<0.05)$. However, there was a significant increase in HDL in the test group when compared with the diabetic rats $(\mathrm{P}<0.05)$. hypercholesteromia and hypertriglyceridemia have been reported to occur in alloxan diabetic rat [14].

A significant increase in serum cholesterol and triglycerides observed in this experiment is in agreement with the findings of [14]. LDL-C is known as a factor in coronary occlusion. Similarly, HDL-C is protective cholesterol and is responsible for transportation of cholesterol from peripheral tissues to the liver.

Considering Laportea aestuans' effect on the lipid components, it can be assumed a potential hypolipidemic agent, which will be a great advantage both in diabetic conditions as well as the associated arteriosclerosis or hyperlipidemic conditions. 


\section{Conclusion}

This study has demonstrated that Laportea aestuans leaves can reverse the hyperglycemia associated with diabetes mellitus. The ability of $L$. aestuans to reduce the level of glycated hemoglobin is a marker showing effective diabetes control and management was also demonstrated.

The study also demonstrated the hypolipidemic effect of $L$. aestuans by reducing the levels of TC, TG, VLDL and LDL. These combined effects can subsequently play a vital role in preventing the incidences of premature occurrence of coronary heart diseases.

This is further strengthened by the increase iin the levels of high density lipoprotein cholesterol levels in the current study. This study suggests that $L$. aestuans leaves might be useful in the treatment of diabetes and cardiovascular diseases caused by hyperlipidemia in humans.

Competing Interests

Authors have declared that no competing interest exists.

\section{References}

[1]. U.A. Essiet, N.I. Edet, and D.N. Bala, "Phytochemical and physiochemical analysis of the leaves of Laportea aestuans (Linn) Chew and Laportea aestuans (Schumach) Chew (male and female)", Asian Journal of Plant Science and Research, vol. 1 no. 2 , pp. $35-42,2011$.

[2]. I.O. Oyewole, and P.F. Akingbala, "Phytochemical analysis and hypolipidemic properties of Jatropha tanjorensis leaf Extract", European Journal of Medicinal Plants, vol. 1, no. 4, pp. 180-185, 2011.

[3]. R.E. Olson, "Discovery of the lipoproteins, their roles in fat transport and their significance as risk factors", J. Nutr., vol. 128, no. 2, pp. $439-443,1998$.

[4]. P. Barter, A.M. Larosa, J.C. Maroni, J. Szareke, M.S.M. Grundy, J.P. Kastelein, and V. Bittner, "HDL cholesterol and cardiovascular events", New Engl. J. Med., vol. 357, no 13, pp. 1301 - 1309, 2007.

[5]. B.N. Okigbo, "Neglected plants of horticultural and nutritional importance in traditional farming systems of tropical Africa". Acta Horticulturae vol. 53, pp. 131-150, 1977.

[6]. Z.O. Gbile and S.K. Adesina, "Nigeria flora and its pharmaceutical potentials". J. Ethnopharmacol, vol. 19, pp. 1-16, 1986.

[7]. W.L. Chew, "A monograph of Laportea (Urticaceae)". Gard. Bull. Singapore, vol. 21, pp. 178 - $195,1969$.

[8]. M. Murray, and J. Pizzorno, Encyclopedia of Natural Medicine, Rockling: Prima Health Publishing, 1997, Pp. 401.

[9]. R.A. Harris, and D.W. Crabbs, Metabolic interrelationship In: Text book of Biochemistry with clinical correlation Ed. Delvin, T.M. New York: John Wiley and Sons Inc., 1982, Pp: 531-559.

[10]. M. Brownlee, "Advanced products of non-enzymatic glycosylation and the pathogensis of diabetic complications. In: Rifkin, H and Porte, Jr. D. Editors. Diabetes mellitus: Theory and practice New York: Elsevier, Pp 279-291. 1990

[11]. B. Arul, K. Kothai, and A.J. Christina, "Hypoglycemia and antihyperglycemic effect of Semearpus anacardium Linn in normal and streptozotocin-induced diabetes rats", Exp. Clin. Pharmacol., vol 26, pp. 759-762, 2004

[12]. O.B. Olayede, "All for love of Nutrients", The seventy eight inaugural lecture of University of Ilorin. University of Ilorin press, Nigeria, Pp: 38 - 39. 2005.

[13]. A. Gupta, R. Gupta, and B. Lal, "Effect of Trigonella foenum-graecum (Fenugreek) seeds on glycemic control and insulin resistance in type 2 diabetes mellitus: a double blind placebo controlled study", J. Assc. Physicians India, vol. 49, pp. 1057 - 1061, 2001.

[14]. S.R. Sharma, S.K. Dwivedi, and R. Swarup, "Hypoglycemic and hypolipidemic effect of Cinnamomum tomala-nees leaves", Ind. J. Exper. Biol., vol. 34, pp. 372 - 374, 1996. 